Supporting Information

\title{
Optically Active Helical Polyacetylene Bearing Ferrocenyl Amino-Acid Derivative in Pendants. Preparation and Application as Chiral Organocatalyst for Asymmetric Aldol Reaction
}

Jinrui Deng, ${ }^{1,2}$ Biao Zhao, ${ }^{1,2}$ Jianping Deng ${ }^{1,2} *$

${ }^{1}$ State Key Laboratory of Chemical Resource Engineering, Beijing University of

Chemical Technology, Beijing 100029, China

${ }^{2}$ College of Materials Science and Engineering, Beijing University of Chemical

Technology, Beijing 100029, China

E-mail: dengjp@mail.buct.edu.cn 


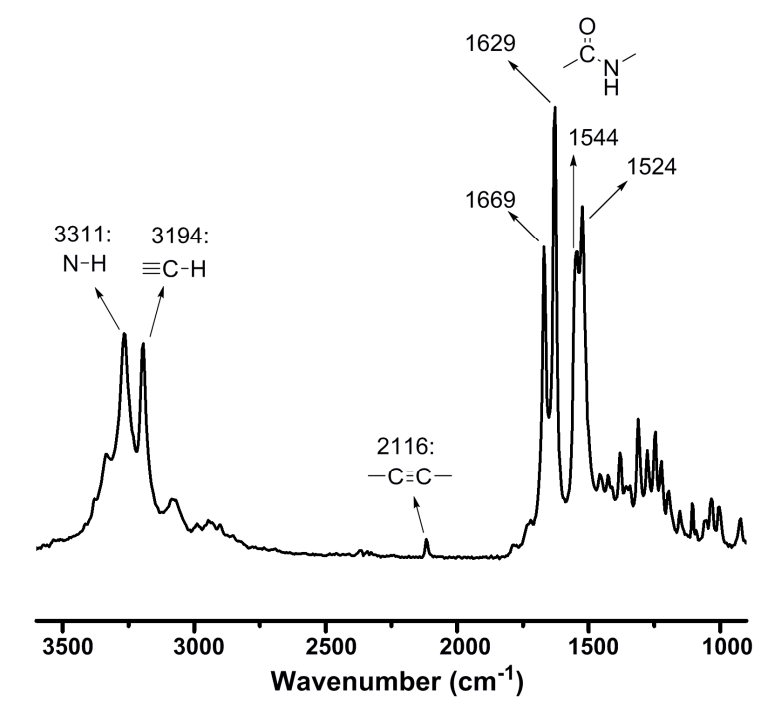

Figure S1. FT-IR spectrum of chiral monomer L-MAFc (in KBr tablet).

IR (KBr): $3311(\mathrm{~N}-\mathrm{H}), 3194(\mathrm{H}-\mathrm{C} \equiv \mathrm{C}), 2116(\mathrm{C} \equiv \mathrm{C})$, 1669, $1544(\mathrm{CONH})$, 1629, 1524 $(\mathrm{CONH}) \mathrm{cm}^{-1}$.

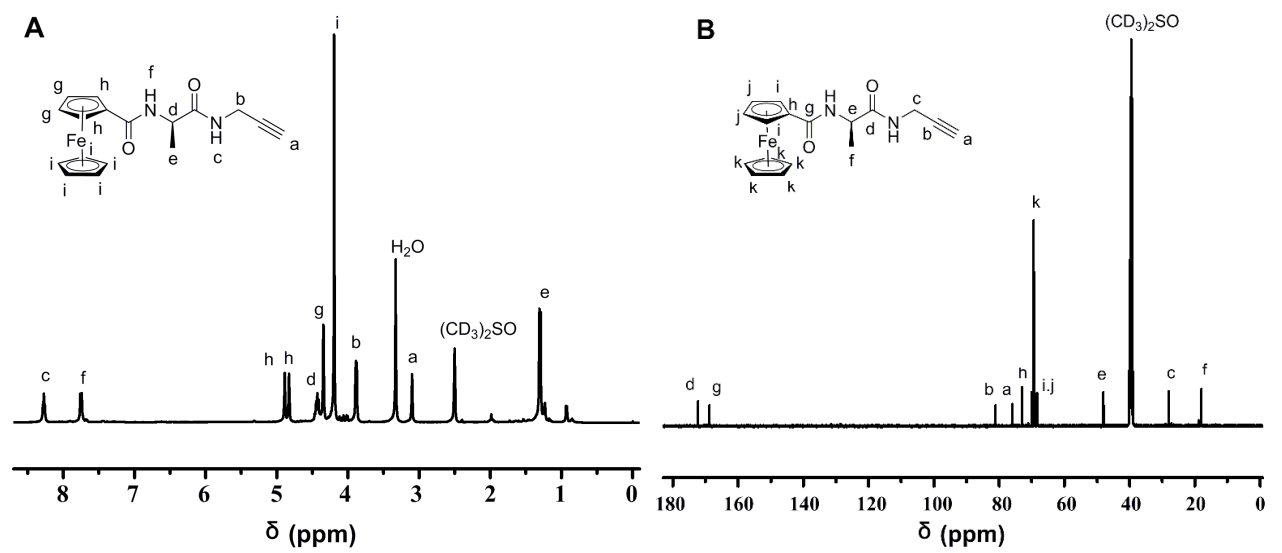

Figure S2. (A) ${ }^{1} \mathrm{H}$ and (B) ${ }^{13} \mathrm{C}$ NMR spectra of L-MAFc; measured in DMSO at $25^{\circ} \mathrm{C}$.

${ }^{1} \mathrm{H}$ NMR $\left(\left[\mathrm{D}_{6}\right] \mathrm{DMSO}\right) \delta=8.28(\mathrm{~d}, J=4.9 \mathrm{~Hz}, 1 \mathrm{H} ; \mathrm{NH}), 7.75(\mathrm{~d}, J=7.8 \mathrm{~Hz}, 1 \mathrm{H} ; \mathrm{NH})$, 4.89 (s, 1H; H-2, Fc), 4.83 (s, 1H; H-5, Fc), 4.43 (dt, $J=14.7,7.2 \mathrm{~Hz}, 1 \mathrm{H} ; \mathrm{CH}), 4.34$

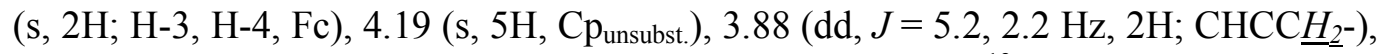
3.10 (s, $\left.1 \mathrm{H} ; \mathrm{C}_{1} \mathrm{CCH}_{2^{-}}\right), 1.30$ (d, $\left.J=7.2 \mathrm{~Hz}, 3 \mathrm{H} ; \mathrm{CH}_{3^{-}}\right) .{ }^{13} \mathrm{C} \mathrm{NMR}\left(\left[\mathrm{D}_{6}\right] \mathrm{DMSO}\right)$ $\delta=172.3(\mathrm{CONH}), 168.8(\mathrm{COFc}), 81.1$ (CHC-), 75.9 (CHC-), 73.0 (C-1, Fc), 69.9 (Cp unsubst. $_{\text {) }}, 69.4$ (C-2, C-5, Fc), 68.3 (C-3, C-4, Fc), $48.0\left(\mathrm{CH}_{3} \mathrm{CH}\right), 28.02\left(\mathrm{CHCCH}_{2}-\right)$, $18.0\left(\mathrm{CH}_{3} \mathrm{CH}\right)$. 


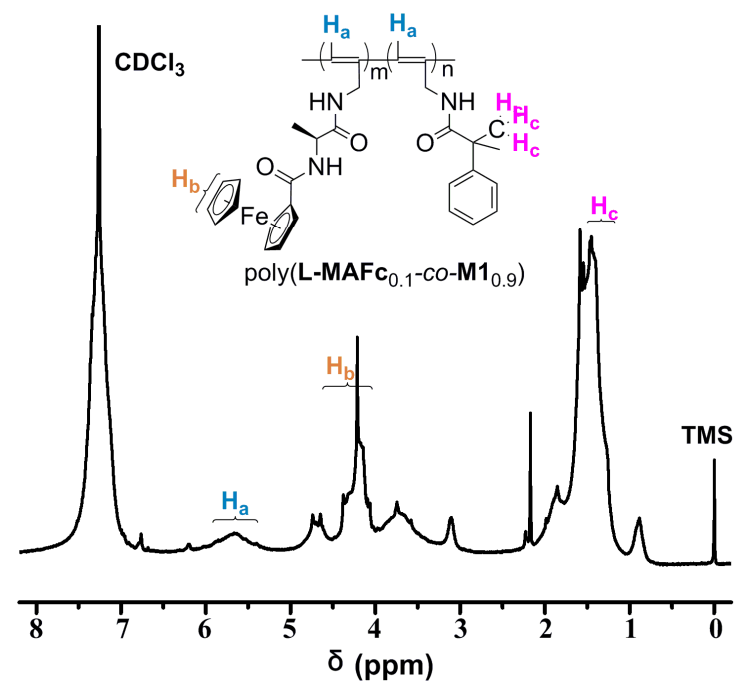

Figure S3. ${ }^{1} \mathrm{H}$ NMR spectrum of poly(L-MAFc $\left.\mathbf{c}_{0.1}-c o-\mathbf{M} \mathbf{1}_{0.9}\right)$; measured in $\mathrm{CDCl}_{3}$ at $25{ }^{\circ} \mathrm{C}$.

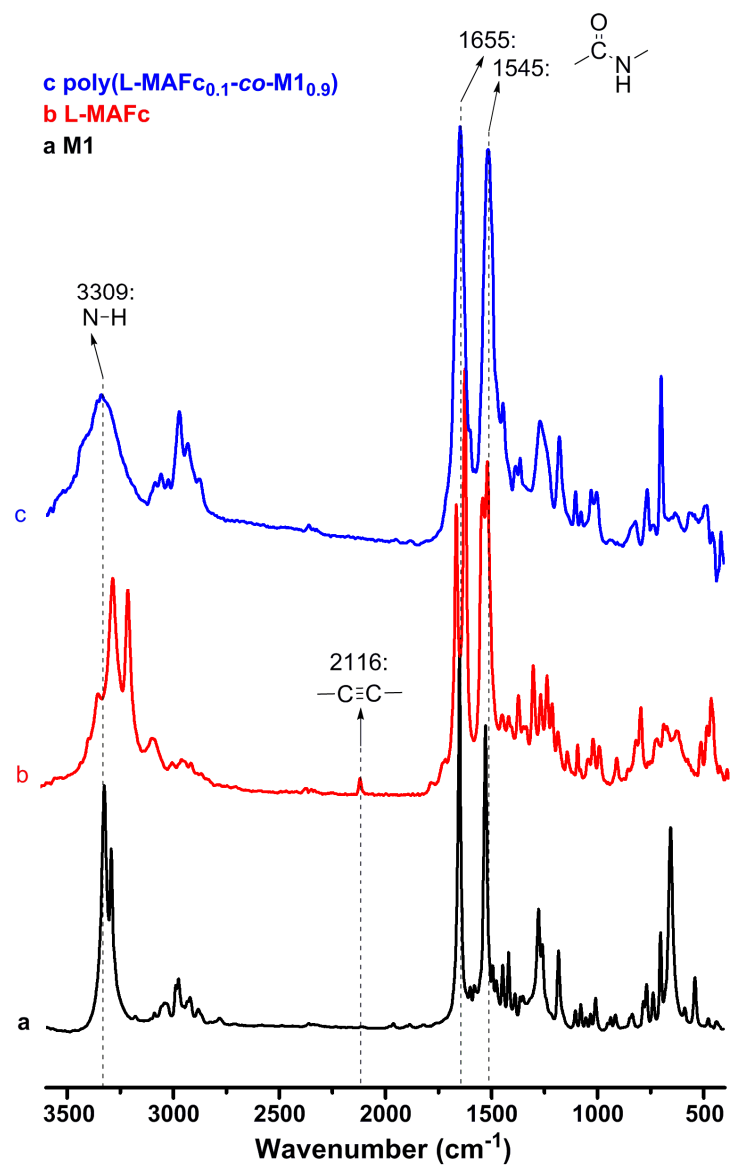

Figure S4. Typical FT-IR spectra of (a) M1, (b) L-MAFc and (c) poly(L-MAFc $\left.\mathbf{c}_{0.1}-\mathrm{co}-\mathbf{M} 1_{0.9}\right)$ (in $\mathrm{KBr}$ tablet). 


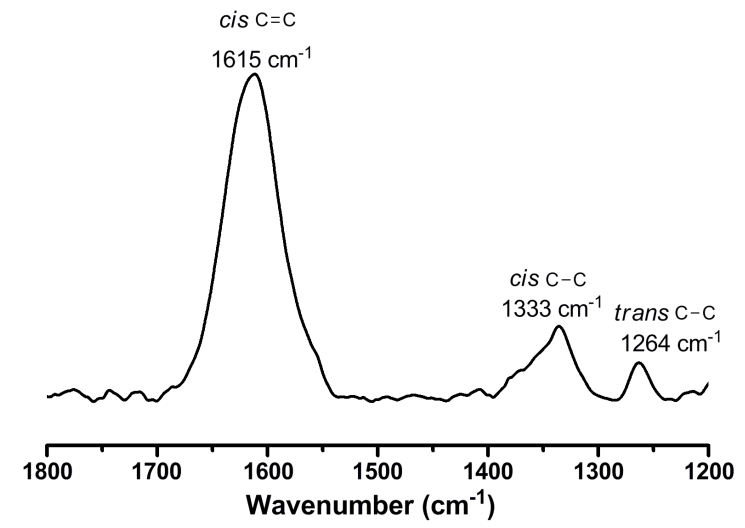

Figure S5. Typical Raman spectrum of poly(L-MAFc $\left.\mathbf{c}_{0.1}-c o-\mathbf{M 1} \mathbf{1}_{0.9}\right)$.
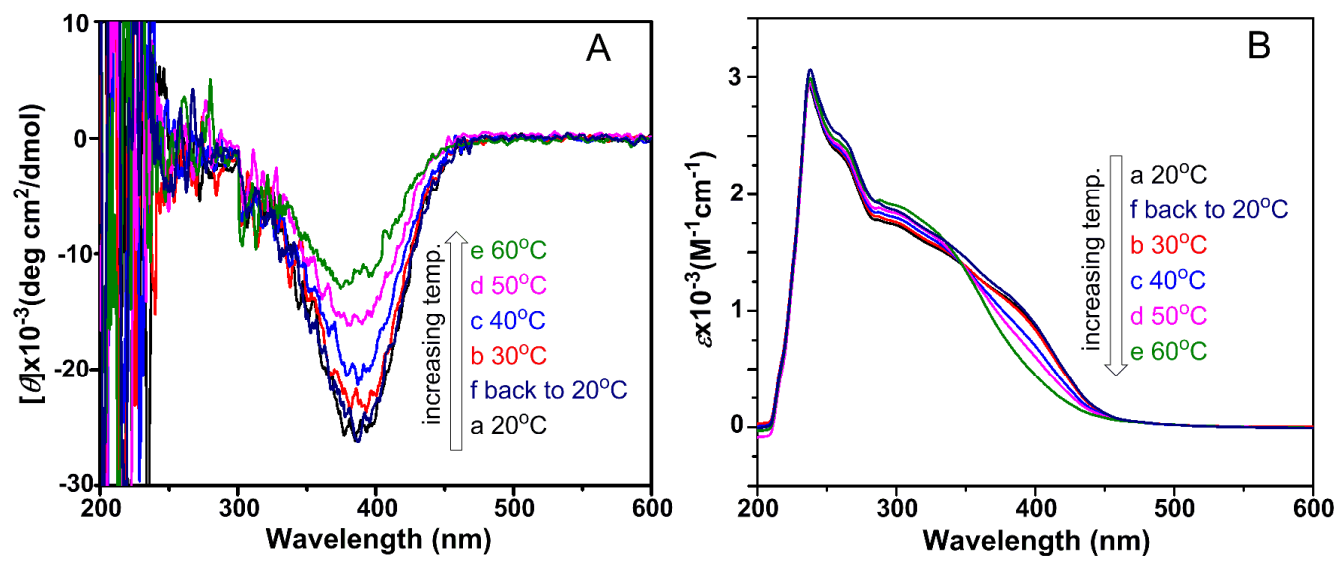

Figure S6. (A) CD and (B) UV-vis spectra of poly(D-MAFr $\left.\mathbf{c}_{0.1}-c o-\mathbf{M} \mathbf{1}_{0.9}\right)$ at increasing temperatures (from 20 to $60{ }^{\circ} \mathrm{C}$ ) in THF $\left(\mathrm{c}=1 \times 10^{-3} \mathrm{~mol} / \mathrm{L}\right)$. 


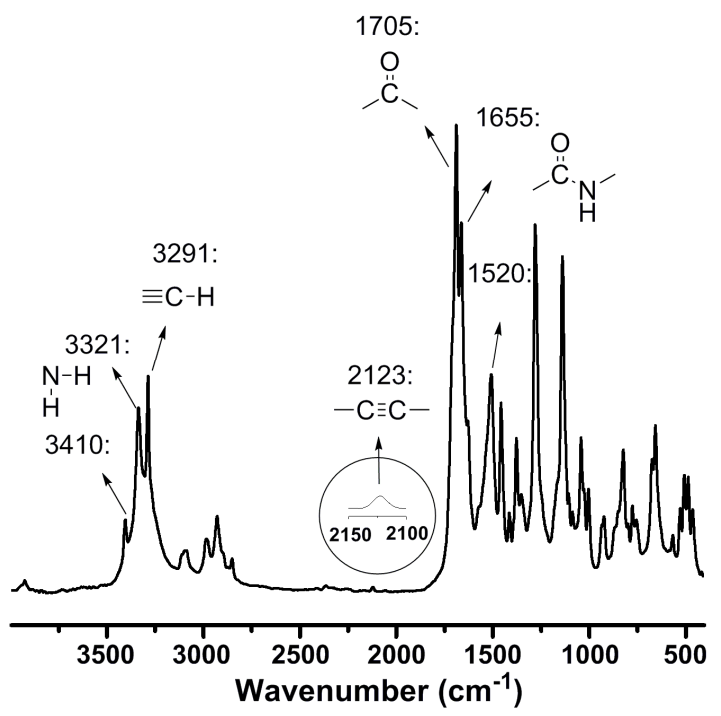

Figure S7. FT-IR spectrum of chiral monomer L-MTFc (in KBr tablet).

IR (KBr): $3410(\mathrm{~N}-\mathrm{H}$ free), $3321(\mathrm{~N}-\mathrm{H}$ assoc.), $3291(\mathrm{H}-\mathrm{C} \equiv \mathrm{C}), 2123(\mathrm{C} \equiv \mathrm{C}), 1705$ $(\mathrm{C}=\mathrm{O}), 1655,1520(\mathrm{CONH}) \mathrm{cm}^{-1}$.
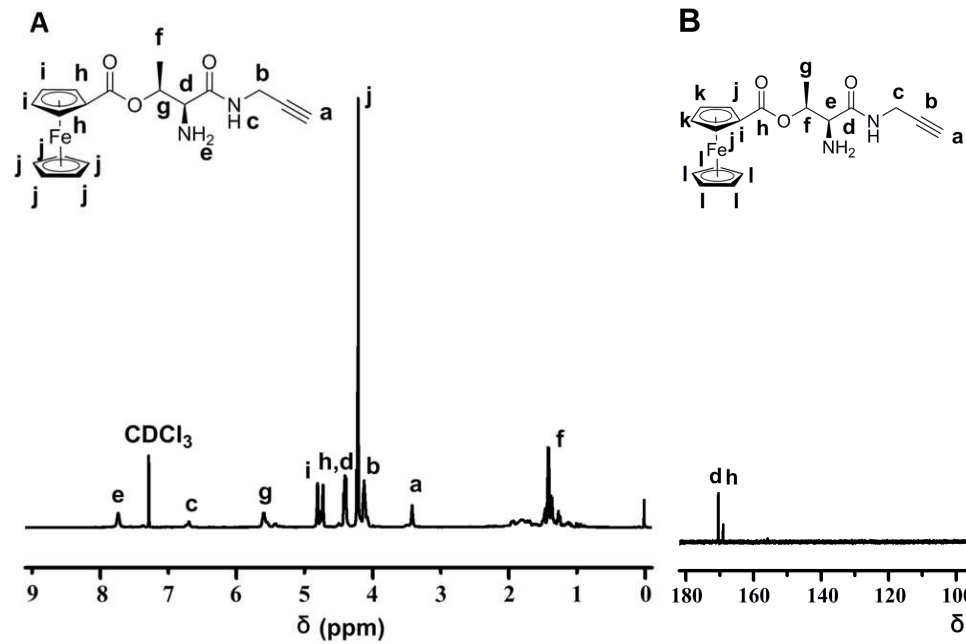

Figure S8. (A) ${ }^{1} \mathrm{H}$ and (B) ${ }^{13} \mathrm{C}$ NMR spectra of L-MTFc; measured in $\mathrm{CDCl}_{3}$ at $25{ }^{\circ} \mathrm{C}$.

${ }^{1} \mathrm{H}$ NMR $\left(\mathrm{CDCl}_{3}\right) \delta=7.74\left(\mathrm{~s}, 2 \mathrm{H} ; \mathrm{NH}_{2}\right), 6.70(\mathrm{~s}, 1 \mathrm{H} ; \mathrm{CONH}), 5.58(\mathrm{t}, J=11.3 \mathrm{~Hz}, 1 \mathrm{H}$; $\mathrm{CH}_{3} \mathrm{CH}-$ ), 4.81 (s, 2H; H-2, H-5, Fc), 4.73 (s, 2H; H-3, H-4, Fc), 4.41 (dt, J= 7.2, 3.8 $\mathrm{Hz}, 1 \mathrm{H} ; \mathrm{CH}$ ), 4.21 (s, 5H; Cp $\mathrm{p}_{\text {unsubst. }}$ ), 4.10 (dd, $J=5.2,2.2 \mathrm{~Hz}, 2 \mathrm{H} ; \mathrm{CHCC} \underline{H}_{2}$ ), 3.42 (s, $\left.1 \mathrm{H} ; \mathrm{C}^{H} \mathrm{CCH}_{2}-\right), 1.14\left(\mathrm{~d}, J=6.1 \mathrm{~Hz}, 3 \mathrm{H} ; \mathrm{CH}_{3}-\right) .{ }^{13} \mathrm{C} \mathrm{NMR}\left(\mathrm{CDCl}_{3}\right) \delta=173.1(\mathrm{CONH})$, 170.4 (COFc), $87.1\left(\mathrm{CHCH}_{3}\right), 79.61\left(\mathrm{CHCCH}_{2}-\right), 76.9$ (C-1, Fc), $), 72.1$ (CHC-), 70.2 (C-2, C-5, Fc), 69.9 (Cp unsubst. $_{\text {) }} 68.3$ (C-3, C-4, Fc), $60.0(\mathrm{CH}), 29.4\left(\mathrm{CHCCH}_{2}-\right), 17.2$ $\left(\mathrm{CH}_{3}-\right)$. 


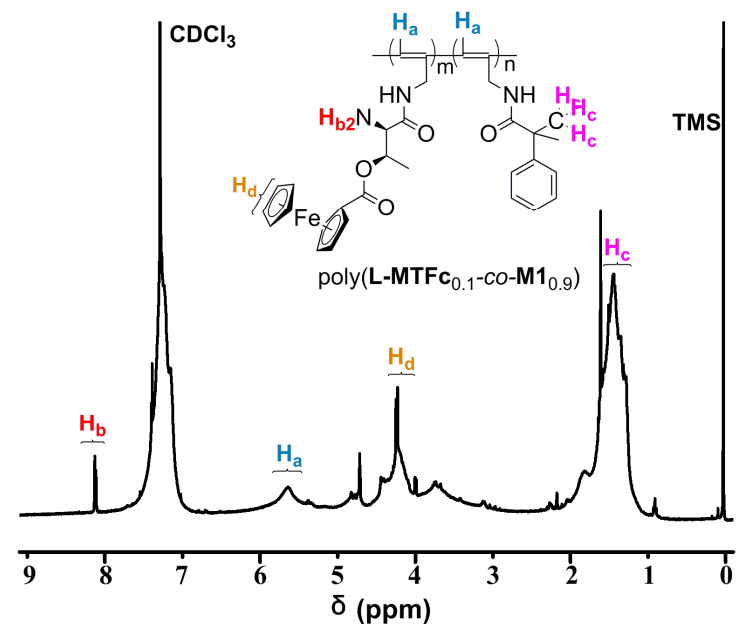

Figure S9. ${ }^{1} \mathrm{H}$ NMR spectrum of poly(L-MTFc $\left.\mathbf{c}_{0.1}-c o-\mathbf{M 1} \mathbf{1}_{0.9}\right)$; measured in $\mathrm{CDCl}_{3}$ at $25^{\circ} \mathrm{C}$. 


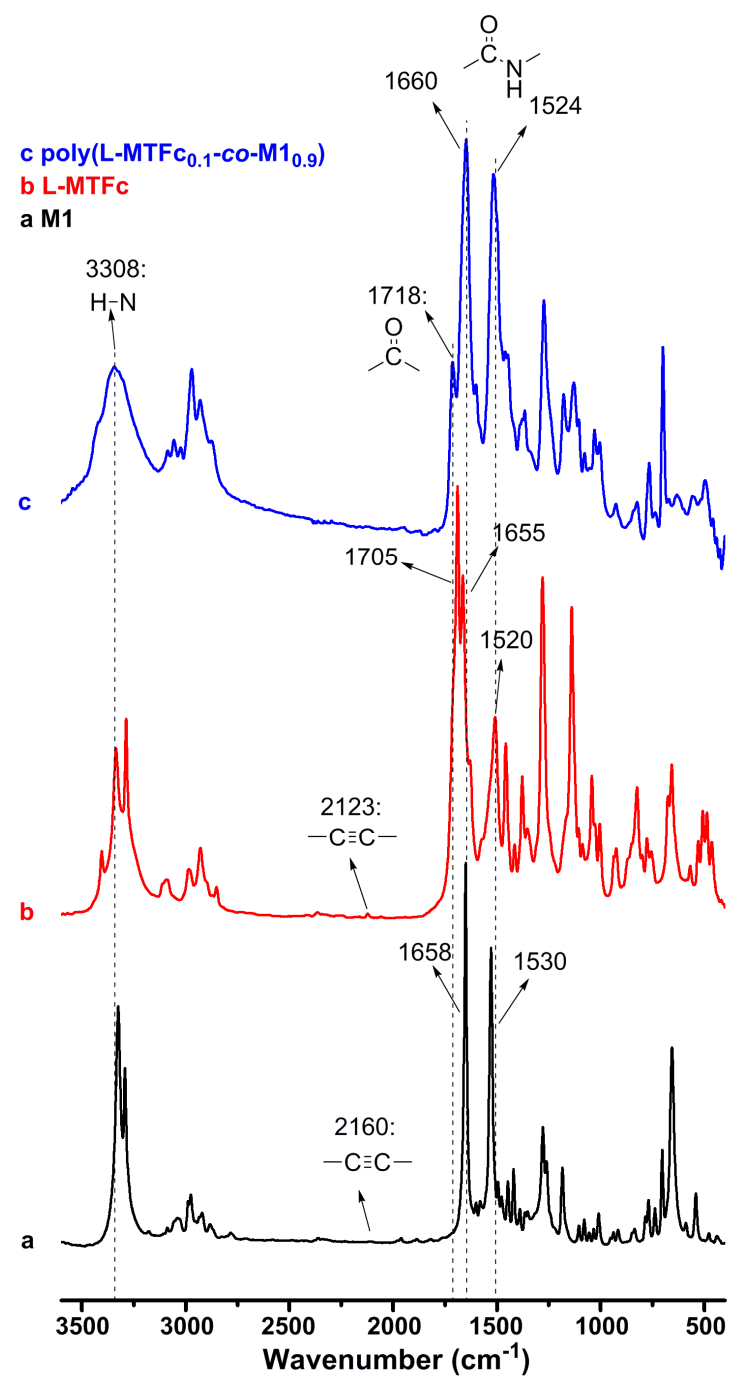

Figure S10. Typical FT-IR spectra of (a) M1, (b) L-MTFc and (c) $\operatorname{poly}\left(\mathbf{L}-\mathbf{M T F c}_{0.1}-\mathrm{co}-\mathbf{M} \mathbf{1}_{0.9}\right)$ (in $\mathrm{KBr}$ tablet).

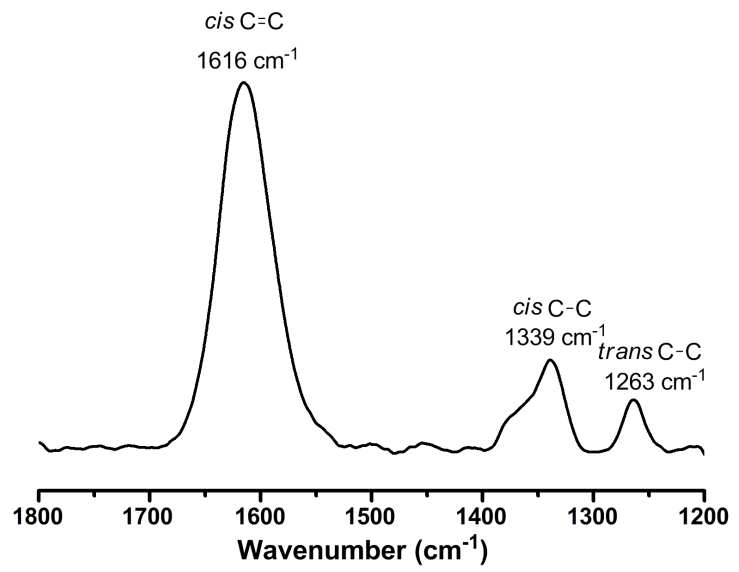

Figure S11. Raman spectrum of poly(L-MTFc $\left.\mathbf{c}_{0.1}-c o-\mathbf{M 1} 1_{0.9}\right)$. 

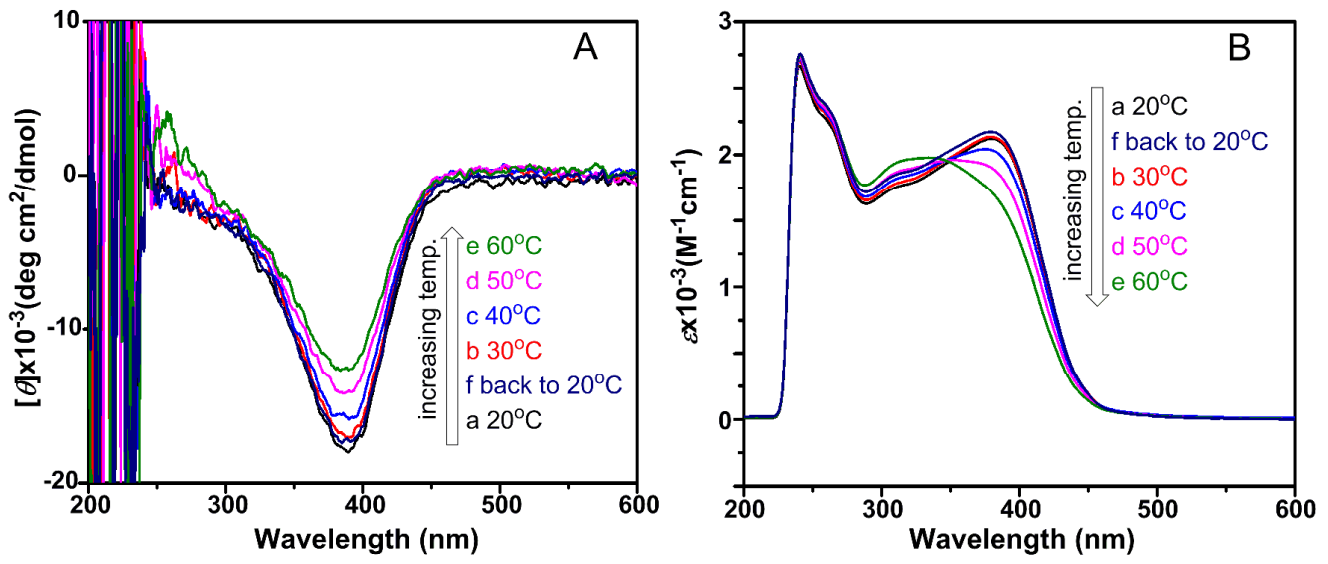

Figure S12. (A) CD and (B) UV-vis spectra of poly(D-MTFe $\left.\mathbf{c}_{0.1}-\mathrm{Co}-\mathbf{M} \mathbf{1}_{0.9}\right)$ at increasing temperatures (from 20 to $\left.60{ }^{\circ} \mathrm{C}\right)$ in THF $\left(\mathrm{c}=1 \times 10^{-3} \mathrm{~mol} / \mathrm{L}\right)$.

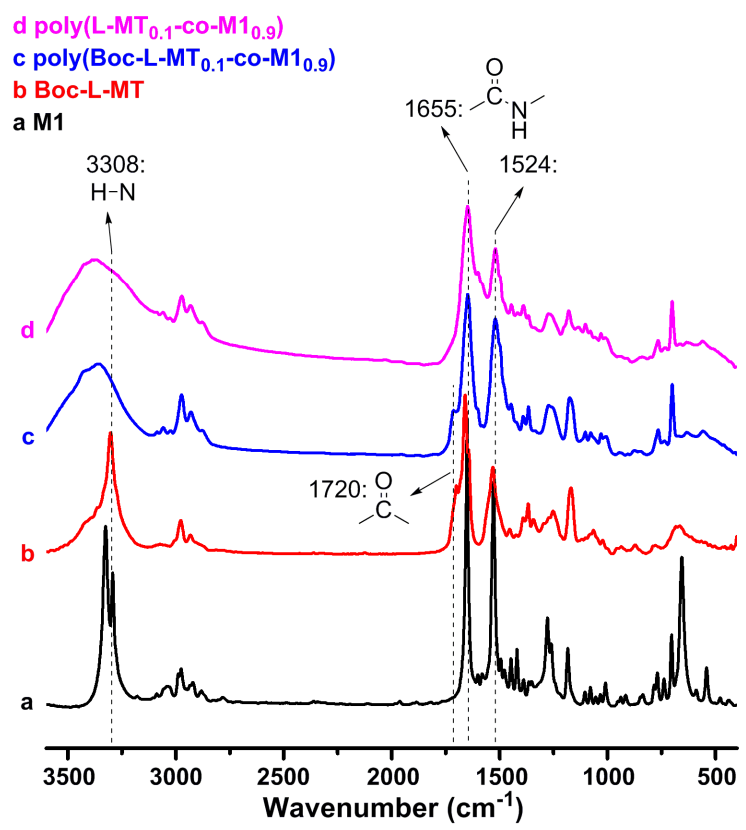

Figure S13. Typical FT-IR spectra of (a) M1, (b) Boc-L-MT and (c) poly(Boc-L-MT $\left.\mathbf{T}_{0.1}-c o-\mathbf{M 1}_{0.9}\right)$ (d) poly(L-MT $\mathbf{T}_{0.1}$-co-M1 $\left.\mathbf{M}_{0.9}\right)$ (in $\mathrm{KBr}$ tablet). 


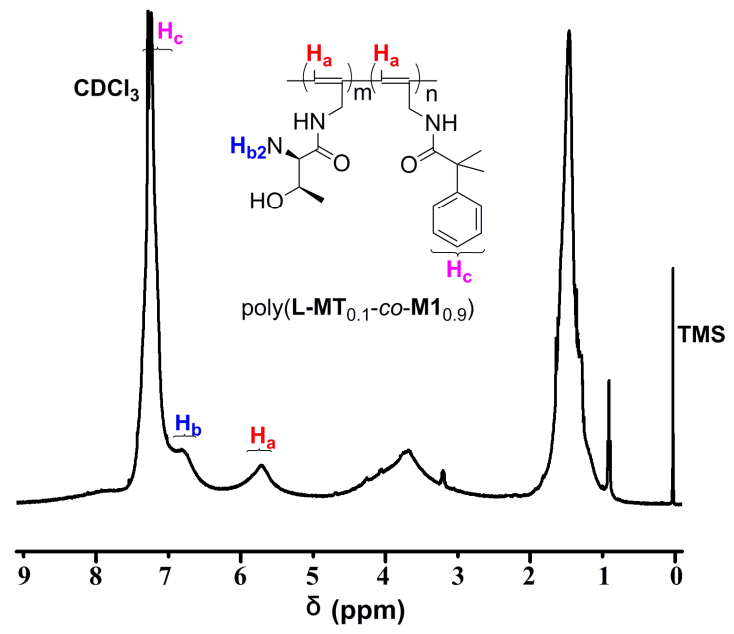

Figure S14. ${ }^{1} \mathrm{H}$ NMR spectrum of poly( $\left.\mathbf{L}-\mathbf{M T} \mathbf{T}_{0.1}-\mathrm{co}-\mathbf{M} \mathbf{1}_{0.9}\right)$; measured in $\mathrm{CDCl}_{3}$ at $25{ }^{\circ} \mathrm{C}$.

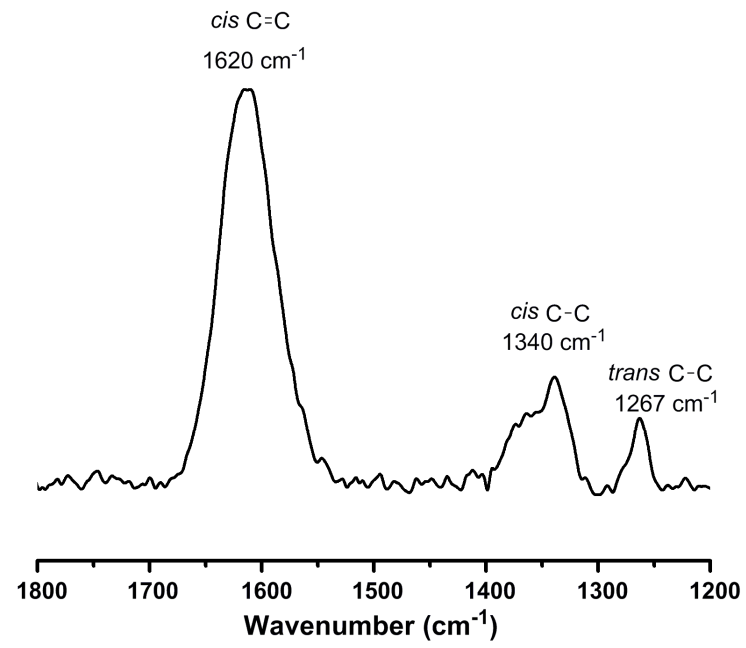

Figure S15. Raman spectrum of poly(L-MT $\left.\mathbf{M}_{0.1}-c o-\mathbf{M} \mathbf{1}_{0.9}\right)$. 

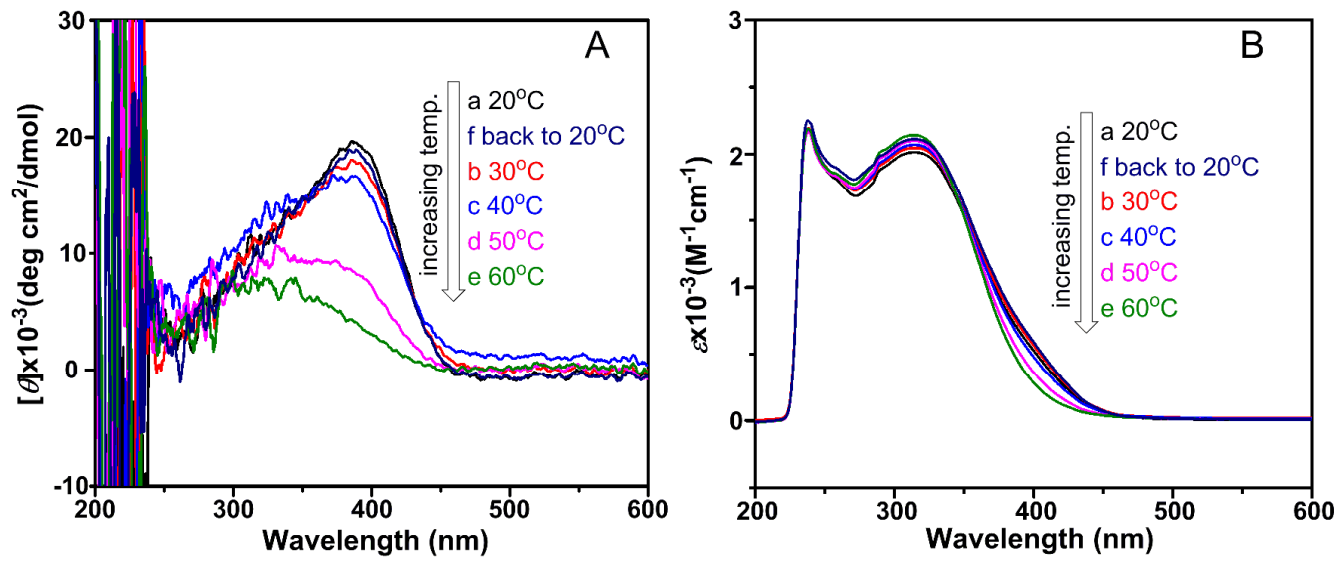

Figure S16. (A) CD and (B) UV-vis spectra of poly(L-MT $\left.\mathbf{T}_{0.1}-c o-\mathbf{M} \mathbf{1}_{0.9}\right)$ at increasing temperatures (from 20 to $60^{\circ} \mathrm{C}$ ) in THF (c $=1 \times 10^{-3} \mathrm{~mol} / \mathrm{L}$ ).

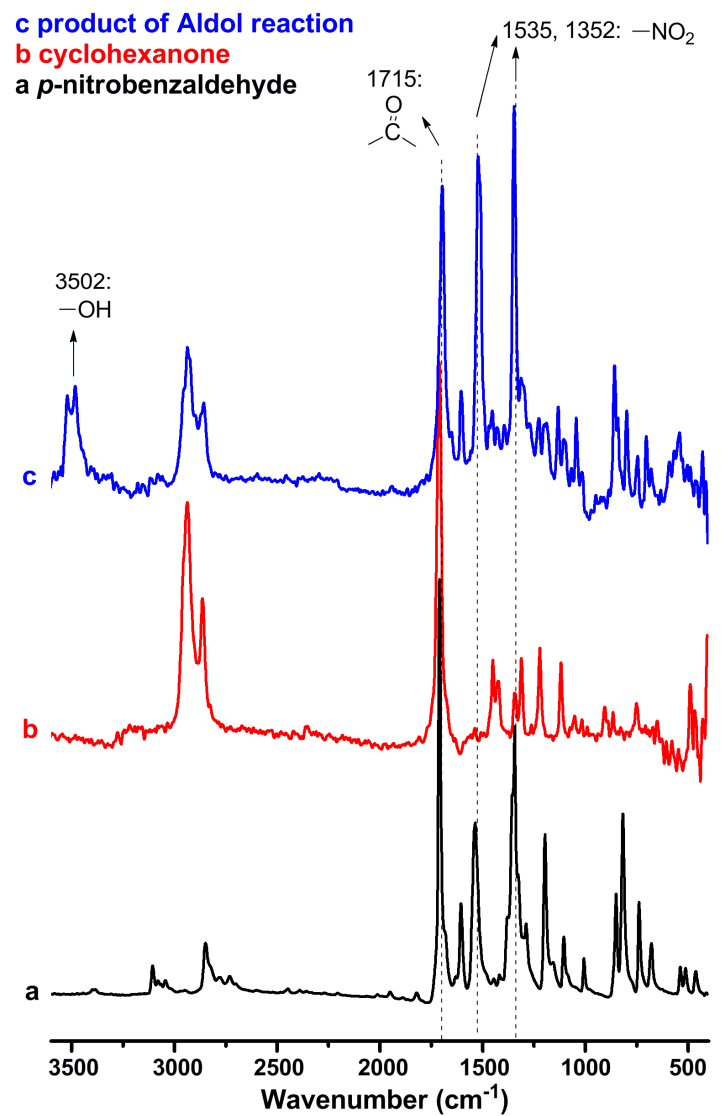

Figure S17. FT-IR spectra of the Aldol reaction product (in $\mathrm{KBr}$ tablet). 

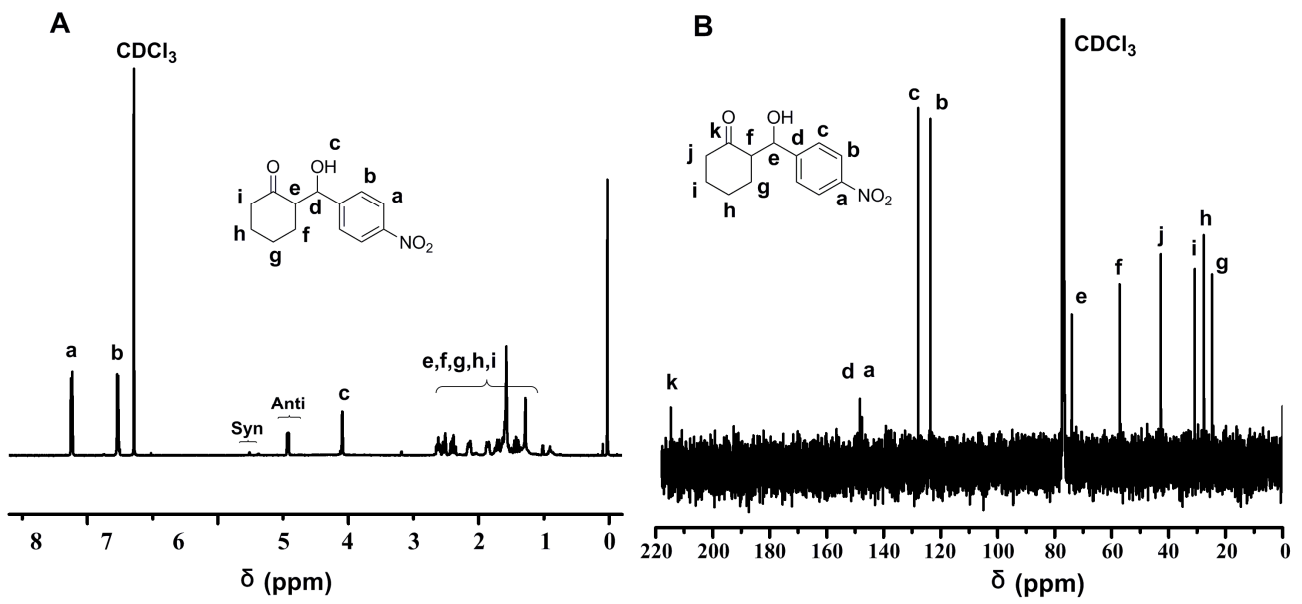

Figure S18. (A) ${ }^{1} \mathrm{H}$ and (B) ${ }^{13} \mathrm{C}$ NMR spectra of the Aldol reaction product; measured in $\mathrm{CDCl}_{3}$ at $25^{\circ} \mathrm{C}$.

(2S, 1'R)-2-(hydroxyl-(p-nitrophenyl) methyl) cyclohexan-1-one: ${ }^{1} \mathrm{H}$ NMR $\left(\mathrm{CDCl}_{3}\right), \delta(\mathrm{ppm}): 8.20-8.21(\mathrm{~d}, 2 \mathrm{H}, \mathrm{J}=8.6), 7.50-7.51(\mathrm{~d}, 2 \mathrm{H}, \mathrm{J}=8.6), 4.89-4.91(\mathrm{dd}$, $1 \mathrm{H}, \mathrm{J}=8.3, \mathrm{~J}=3.2), 4.05-4.06(\mathrm{~d}, 1 \mathrm{H}, \mathrm{J}=3.2), 2.57-2.61(\mathrm{~m}, 1 \mathrm{H}), 2.48-2.52(\mathrm{~m}$, 1H), 2.34-2.39 (m, 1H), 2.09-2.14 (m, 1H), 1.82-1.84 (m, 1H), 1.63-1.71(m, 1H), $1.52-1.61(\mathrm{~m}, 2 \mathrm{H}), 1.35-1.42(\mathrm{~m}, 1 \mathrm{H}) .{ }^{13} \mathrm{C} \mathrm{NMR}\left(\mathrm{CDCl}_{3}\right), \delta(\mathrm{ppm}): 214.8,148.4$, 147.6, 127.9, 123.6, 74.0, 57.2, 42.7, 30.8, 27.7, 24.7.

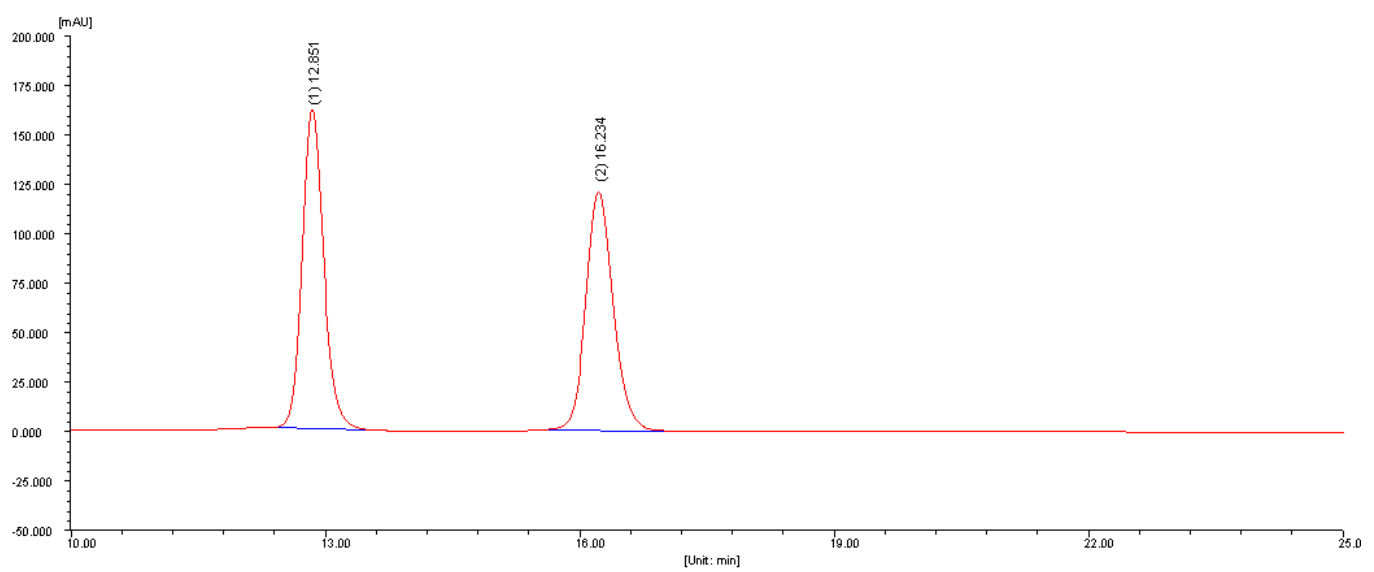

Figure S19. Typical HPLC spectrum of the racemic Aldol reaction product. 


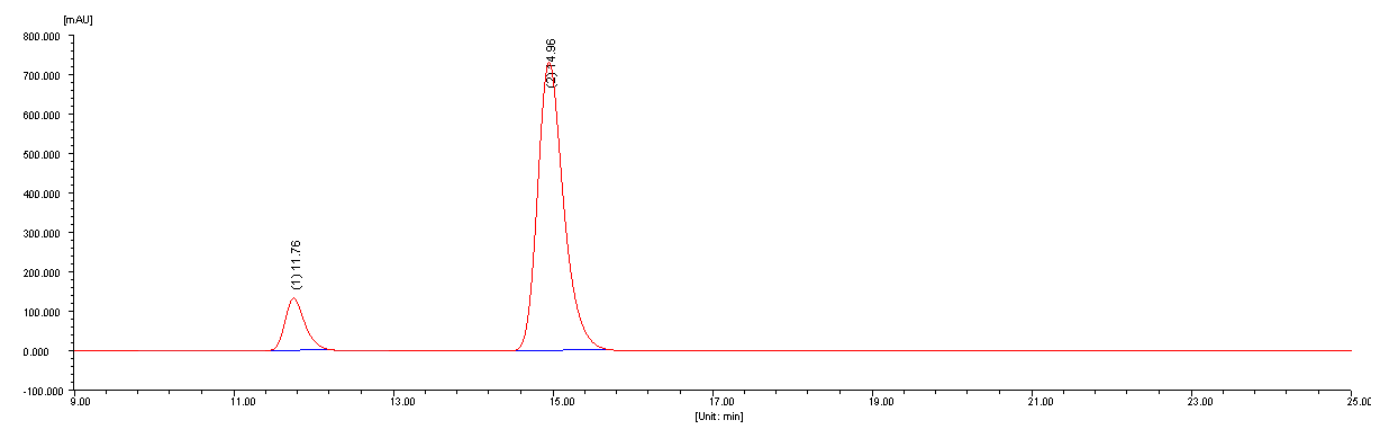

Figure S20. Typical HPLC spectrum of the Aldol reaction product catalyzed by $\operatorname{poly}\left(\mathbf{L}-\mathbf{M T} \mathbf{T}_{0.1}-\mathrm{co}-\mathbf{M} \mathbf{1}_{0.9}\right)$.

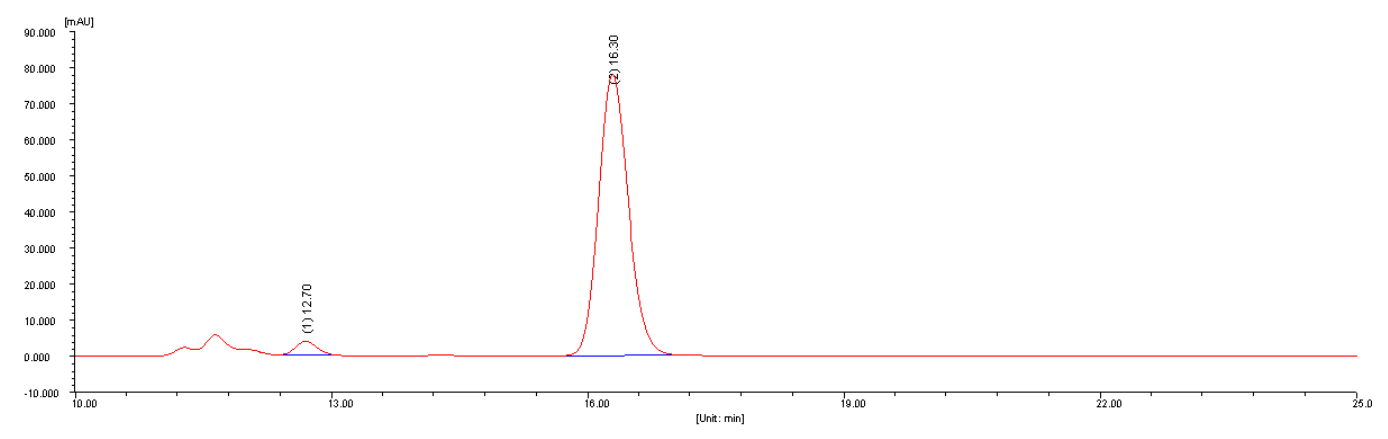

Figure S21. Typical HPLC spectrum of the Aldol reaction product catalyzed by $\operatorname{poly}\left(\mathbf{L}-\mathbf{M T F e}_{0.1}-c o-\mathbf{M 1} \mathbf{1}_{0.9}\right)$ in the presence of $\mathrm{PhCOOH}\left(0.1\right.$ equiv.) and $\mathrm{H}_{2} \mathrm{O}$ (10 equiv.).

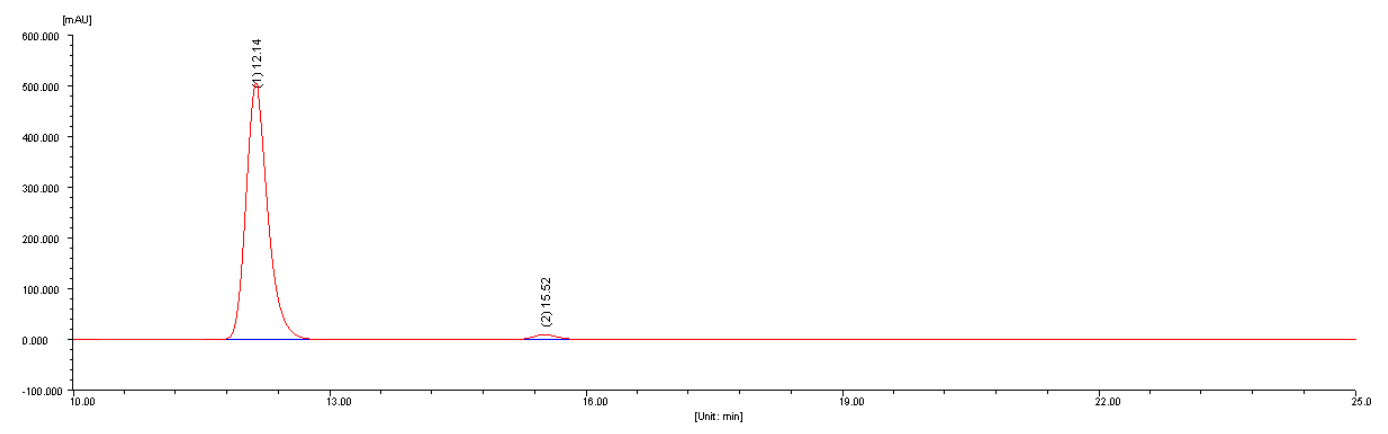

Figure S22. Typical HPLC spectrum of the Aldol reaction product catalyzed by poly(D-MTF $\left.\mathbf{c}_{0.1}-c o-\mathbf{M 1} \mathbf{1}_{0.9}\right)$ in the presence of $\mathrm{PhCOOH}\left(0.1\right.$ equiv.) and $\mathrm{H}_{2} \mathrm{O}$ (10 equiv.). 